\title{
A stochastic prey-predator model with time-dependent delays
}

\section{Xiangjun Dai", Zhi Mao and Xiaojun Li}

"Correspondence: daiaga0921@126.com School of Data Science, TongRen University, Tongren, 554300, P.R China

\begin{abstract}
A stochastic predator-prey system with time-dependent delays is considered. Firstly, we show the existence of a global positive solution and stochastically ultimate boundedness. Secondly, the critical value between weak persistence and extinction of the prey is obtained and we also give the asymptotic pathwise estimation. Finally, we simulate the model to illustrate our results.
\end{abstract}

MSC: 92B05; 92D25; 93E03

Keywords: prey-predator system; time-dependent delays; stochastically ultimate bounded; persistence; extinction

\section{Introduction}

During the last decades, the classical predator-prey system has gained an important theoretical and practical significance, which has been studied extensively, and many excellent results concerning the permanence, extinction and global attractive of the predator-prey system have been obtained; see [1-5].

A classical two-species predator-prey system can be expressed as follows:

$$
\left\{\begin{array}{l}
\frac{d x(t)}{d t}=x(t)\left[r-a_{11} x(t)-a_{12} y(t)\right] \\
\frac{d y(t)}{d t}=y(t)\left[-d+a_{21} x(t)-a_{22} y(t)\right]
\end{array}\right.
$$

where $x(t)$ and $y(t)$ stand for the population sizes of the prey and the predator, respectively, and $r, d, a_{i j}(i, j=1,2)$ are positive constants. For biological interpretation of each coefficient in system (1), we refer the reader to [1].

On the one hand, more realistic and interesting models of population interactions should take the effects of time delay into account [6-8], such as their maturation time. In consideration of the fact that time delays are not resistant to time fluctuations [9-11], system (1) becomes

$$
\left\{\begin{array}{l}
\frac{d x(t)}{d t}=x(t)\left[r-a_{11} x(t)-a_{12} x\left(t-\tau_{1}(t)\right)-a_{13} y\left(t-\tau_{2}(t)\right)\right], \\
\frac{d y(t)}{d t}=y(t)\left[-d+a_{21} x\left(t-\tau_{3}(t)\right)-a_{22} y(t)-a_{23} y\left(t-\tau_{4}(t)\right)\right],
\end{array}\right.
$$

(c) The Author(s) 2017. This article is distributed under the terms of the Creative Commons Attribution 4.0 International License (http://creativecommons.org/licenses/by/4.0/), which permits unrestricted use, distribution, and reproduction in any medium, provided you give appropriate credit to the original author(s) and the source, provide a link to the Creative Commons license, and indicate if changes were made. 
where $a_{13}>0, a_{23}>0$ and $\tau_{i}(t)(i=1,2,3,4)$ is a nonnegative, bounded and differentiable function on $[0,+\infty)$, and satisfies

$$
\bar{\tau}=\max _{i=1,2,3,4} \sup _{t \geq 0}\left\{\tau_{i}(t)\right\} \geq 0 .
$$

$\tau_{i}^{\prime}(t)(i=1,2,3,4)$ are continuous bounded functions on $[0,+\infty)$ with $\tau_{i}^{\prime}(t)=\frac{d \tau_{i}(t)}{d t}$.

On the other hand, population systems are inevitably subject to the environmental noises in the natural environment (see [12-15]). May [16] pointed out that, due to environmental fluctuations, the birth rate, carrying capacity, competition coefficients and other parameters involved in the system exhibit random fluctuation to a greater or lesser extent (see [17-20]). We suppose that the white noise affects all parameters. We usually estimate a value by an average value plus an error term. By the central limit theorem, the error term follows a normal distribution, thus, we can replace each growth rate by an average rate plus an error term, $r \rightarrow r+\sigma_{11} \dot{B}_{11}(t)$, and similarly, $-d \rightarrow-d+\sigma_{21} \dot{B}_{21}(t), a_{i j} \rightarrow a_{i j}+\sigma_{i(j+1)} \dot{B}_{i(j+1)}(t)$, where $\sigma_{i j}^{2}$ denotes the intensity of the noise and $\dot{B}_{i(j+1)(t)}$ is a standard white noise, as $B_{i j}(t)$ is a standard Brownian motion defined on a complete probability space $\left(\Omega, \mathcal{F},\left\{\mathcal{F}_{t}\right\}_{t \geq 0}, P\right)$ with a filtration $\left\{\mathcal{F}_{t}\right\}_{t \geq 0}$ satisfying the usual conditions. Then this system will become the following stochastic autonomous predator-prey system with time-dependent delays:

$$
\left\{\begin{aligned}
d x(t)= & x(t)\left[r-a_{11} x(t)-a_{12} x\left(t-\tau_{1}(t)\right)-a_{13} y\left(t-\tau_{2}(t)\right)\right] d t+\sigma_{11} x(t) d B_{11}(t) \\
& +\sigma_{12} x^{2}(t) d B_{12}(t)+\sigma_{13} x(t) x\left(t-\tau_{1}(t)\right) d B_{13}(t)+\sigma_{14} x(t) y\left(t-\tau_{2}(t)\right) d B_{14}(t) \\
d y(t)= & y(t)\left[-d+a_{21} x\left(t-\tau_{3}(t)\right)-a_{22} y(t)-a_{23} y\left(t-\tau_{4}(t)\right)\right] d t+\sigma_{21} y(t) d B_{21}(t) \\
& +\sigma_{22} y(t) x\left(t-\tau_{3}(t)\right) d B_{22}(t)+\sigma_{23} y^{2}(t) d B_{23}(t)+\sigma_{24} y(t) y\left(t-\tau_{4}(t)\right) d B_{24}(t)
\end{aligned}\right.
$$

with initial conditions $x(\theta)=\varphi_{1}(\theta)>0, y(\theta)=\varphi_{2}(\theta)>0, \theta \in[-\bar{\tau}, 0]$, where $\varphi_{i}(\theta)$ is a continuous function on $[-\bar{\tau}, 0]$.

For simplicity, we introduce the following notations here:

$$
\begin{aligned}
& R_{+}^{n}=\left\{\left(a_{1}, a_{2}, \ldots, a_{n}\right) \in R^{n}: a_{i}>0, i=1,2, \ldots, n\right\}, \\
& \langle f(t)\rangle=\frac{1}{t} \int_{0}^{t} f(s) d s, \quad f^{*}=\limsup _{t \rightarrow+\infty} f(t), \quad f_{*}=\liminf _{t \rightarrow+\infty} f(t) .
\end{aligned}
$$

Throughout this paper, the delay functions satisfy the following condition $\tau^{\prime}<1$, where $\tau^{\prime}=\max _{i=1,2,3,4} \sup _{t \geq 0} \tau_{i}^{\prime}(t)$.

\section{Positive and global solution}

Since $x(t)$ and $y(t)$ in system (2) represent population sizes at time $t$, they must be nonnegative, so, for further study, the first thing is to prove the solution of system (2) has a unique global positive solution.

Lemma 2.1 For any given initial value $\left(\varphi_{1}(\theta), \varphi_{2}(\theta)\right) \in C\left([-\bar{\tau}, 0], R_{+}^{2}\right)$, system (2) has a unique global positive solution $(x(t), y(t))$ on $t \geq-\bar{\tau}$ and the solution will remain in $R_{+}^{2}$ with probability 1.

Proof Since the coefficients of system (2) are locally Lipschitz continuous, for any given initial value $\left(\varphi_{1}(\theta), \varphi_{2}(\theta)\right) \in C\left([-\bar{\tau}, 0], R_{+}^{2}\right)$, there exists a local positive solution $(x(t), y(t))$ 
on $t \in\left[-\bar{\tau}, \tau_{e}\right)$, where $\tau_{e}$ denotes the explosion time. To verify that this solution is global, we only need to prove $\tau_{e}=+\infty$ a.s. The proof is similar to [17] by defining functions

$$
\begin{aligned}
V_{1}(x, y)= & \sqrt{x}-1-\ln \sqrt{x}+\sqrt{y}-1-\ln \sqrt{y} \\
V_{2}(x, y)= & \frac{1+\sigma_{13}^{2}}{4\left(1-\tau^{\prime}\right)} \int_{t-\tau_{1}(t)}^{t} x^{2}\left(s-\tau_{1}(s)\right) d s+\frac{1+\sigma_{22}^{2}}{4\left(1-\tau^{\prime}\right)} \int_{t-\tau_{3}(t)}^{t} x^{2}\left(s-\tau_{3}(s)\right) d s \\
& +\frac{1+\sigma_{14}^{2}}{4\left(1-\tau^{\prime}\right)} \int_{t-\tau_{2}(t)}^{t} y^{2}\left(s-\tau_{2}(s)\right) d s+\frac{1+\sigma_{24}^{2}}{4\left(1-\tau^{\prime}\right)} \int_{t-\tau_{4}(t)}^{t} y^{2}\left(s-\tau_{4}(s)\right) d s .
\end{aligned}
$$

Applying Itô's formula to $V(x, y)=V_{1}(x, y)+V_{2}(x, y)$, we have

$$
\begin{aligned}
d V(x, y)= & L V_{1}(x, y) d t+L V_{2}(x, y) d t+\frac{(\sqrt{x}-1) \sigma_{11}}{2} d B_{11}(t)+\frac{\sigma_{12}(\sqrt{x}-1) x}{2} d B_{12}(t) \\
& +\frac{\sigma_{13}(\sqrt{x}-1) x\left(t-\tau_{1}(t)\right)}{2} d B_{13}(t)+\frac{\sigma_{14}(\sqrt{x}-1) y\left(t-\tau_{2}(t)\right)}{2} d B_{14}(t) \\
& +\frac{\sigma_{21}(\sqrt{y}-1)}{2} d B_{21}(t)+\frac{\sigma_{23}(\sqrt{y}-1) y}{2} d B_{23}(t) \\
& +\frac{\sigma_{22}(\sqrt{y}-1) y\left(t-\tau_{3}(t)\right)}{2} d B_{22}(t)+\frac{\sigma_{24}(\sqrt{y}-1) y\left(t-\tau_{4}(t)\right)}{2} d B_{24}(t),
\end{aligned}
$$

where

$$
\begin{aligned}
L V_{1}(x, y)= & \frac{(\sqrt{x}-1)}{2}\left[r-a_{11} x-a_{12} x\left(t-\tau_{1}(t)\right)-a_{13} y\left(t-\tau_{2}(t)\right)\right]+\frac{(2-\sqrt{x}) \sigma_{11}^{2}}{8} \\
& +\frac{(2-\sqrt{x}) \sigma_{12}^{2} x^{2}}{8}+\frac{(2-\sqrt{x}) \sigma_{13}^{2} x\left(t-\tau_{1}(t)\right)^{2}}{8}+\frac{(2-\sqrt{x}) \sigma_{14}^{2} y\left(t-\tau_{2}(t)\right)^{2}}{8} \\
& +\frac{(\sqrt{y}-1)}{2}\left[-d+a_{21} x\left(t-\tau_{3}(t)\right)-a_{22} y-a_{23} y\left(t-\tau_{4}(t)\right)\right]+\frac{(2-\sqrt{y}) \sigma_{21}^{2}}{8} \\
& +\frac{(2-\sqrt{y}) \sigma_{22}^{2} x\left(t-\tau_{3}(t)\right)^{2}}{8}+\frac{(2-\sqrt{y}) \sigma_{23}^{2} y^{2}}{8}+\frac{(2-\sqrt{y}) \sigma_{24}^{2} y\left(t-\tau_{4}(t)\right)^{2}}{8} \\
\leq & 0.5 r \sqrt{x}-0.5 a_{11} x^{1.5}+0.5 a_{11} x+0.25\left(1+\sigma_{13}^{2}\right) x^{2}\left(t-\tau_{1}(t)\right) \\
& +0.25 a_{12}^{2}(\sqrt{x}-1)^{2}+0.25 a_{13}^{2}(\sqrt{x}-1)^{2}+0.25\left(1+\sigma_{14}^{2}\right) y^{2}\left(t-\tau_{2}(t)\right) \\
& +0.125(2-\sqrt{x}) \sigma_{11}^{2}+0.25 \sigma_{12}^{2} x^{2}-0.125 \sigma_{12}^{2} x^{2.5}+0.5 d+0.25 a_{21}^{2}(\sqrt{y}-1) \\
& +0.25\left(1+\sigma_{22}^{2}\right) x^{2}\left(t-\tau_{3}(t)\right)-0.5 a_{22} y^{1.5}+0.5 a_{22}+0.25 a_{23}^{2}(\sqrt{y}-1)^{2} \\
& +0.25\left(1+\sigma_{24}^{2}\right) y^{2}\left(t-\tau_{4}(t)\right)+0.125(2-\sqrt{y}) \sigma_{21}^{2}+0.25 \sigma_{23}^{2} y^{2}-0.125 \sigma_{23}^{2} y^{2.5},
\end{aligned}
$$

because $\tau^{\prime}<1$, we have

$$
\begin{aligned}
L V_{2}(x, y)= & \frac{1+\sigma_{13}^{2}}{4\left(1-\tau^{\prime}\right)} x^{2}(t)-\frac{\left(1-\tau_{1}^{\prime}(t)\right)\left(1+\sigma_{13}^{2}\right)}{4\left(1-\tau^{\prime}\right)} x^{2}\left(t-\tau_{1}(t)\right)+\frac{1+\sigma_{22}^{2}}{4\left(1-\tau^{\prime}\right)} x^{2}(t) \\
& -\frac{\left(1-\tau_{3}^{\prime}(t)\right)\left(1+\sigma_{22}^{2}\right)}{4\left(1-\tau^{\prime}\right)} x^{2}\left(t-\tau_{3}(t)\right)-\frac{\left(1-\tau_{2}^{\prime}(t)\right)\left(1+\sigma_{22}^{2}\right)}{4\left(1-\tau^{\prime}\right)} y^{2}\left(t-\tau_{2}(t)\right) \\
& +\frac{1+\sigma_{22}^{2}}{4\left(1-\tau^{\prime}\right)} y^{2}(t)+\frac{1+\sigma_{24}^{2}}{4\left(1-\tau^{\prime}\right)} y^{2}(t)-\frac{\left(1-\tau_{4}^{\prime}(t)\right)\left(1+\sigma_{24}^{2}\right)}{4\left(1-\tau^{\prime}\right)} y^{2}\left(t-\tau_{4}(t)\right)
\end{aligned}
$$




$$
\begin{aligned}
\leq & \frac{1+\sigma_{13}^{2}}{4\left(1-\tau^{\prime}\right)} x^{2}(t)-\frac{1+\sigma_{13}^{2}}{4} x^{2}\left(t-\tau_{1}(t)\right)+\frac{1+\sigma_{22}^{2}}{4\left(1-\tau^{\prime}\right)} x^{2}(t)-\frac{1+\sigma_{22}^{2}}{4} x^{2}\left(t-\tau_{3}(t)\right) \\
& +\frac{1+\sigma_{14}^{2}}{4\left(1-\tau^{\prime}\right)} y^{2}(t)-\frac{1+\sigma_{14}^{2}}{4} y^{2}\left(t-\tau_{2}(t)\right)+\frac{1+\sigma_{24}^{2}}{4\left(1-\tau^{\prime}\right)} y^{2}(t) \\
& -\frac{1+\sigma_{24}^{2}}{4} y^{2}\left(t-\tau_{4}(t)\right),
\end{aligned}
$$

then

$$
\begin{aligned}
L V(x, y)= & L V_{1}(x, y)+L V_{2}(x, y) \\
= & 0.5 r \sqrt{x}-0.5 a_{11} x^{1.5}+0.5 a_{11} x+\frac{1+\sigma_{13}^{2}}{4\left(1-\tau^{\prime}\right)} x^{2}+0.25 a_{12}^{2}(\sqrt{x}-1)^{2} \\
& +0.25 a_{13}^{2}(\sqrt{x}-1)^{2}+\frac{1+\sigma_{14}^{2}}{4\left(1-\tau^{\prime}\right)} y^{2}+0.125(2-\sqrt{x}) \sigma_{11}^{2}+0.25 \sigma_{12}^{2} x^{2} \\
& -0.125 \sigma_{12}^{2} x^{2.5}+0.5 d+0.25 a_{21}^{2}(\sqrt{y}-1)+\frac{1+\sigma_{22}^{2}}{4\left(1-\tau^{\prime}\right)} x^{2}-0.5 a_{22} y^{1.5} \\
& +0.5 a_{22}+0.25 a_{23}^{2}(\sqrt{y}-1)^{2}+\frac{1+\sigma_{24}^{2}}{4\left(1-\tau^{\prime}\right)} y^{2}(t)+0.125(2-\sqrt{y}) \sigma_{21}^{2} \\
& +0.25 \sigma_{2}^{2} y^{2}-0.125 \sigma_{2}^{2} y^{2.5} \\
\leq & K .
\end{aligned}
$$

There is a positive constant $K$ satisfying the above inequality. By a similar proof to [17], we can obtain the desired assertion, and it is omitted in here.

\section{Stochastically ultimate boundedness}

Definition 3.1 (See [20]) The solution $X(t)=(x(t), y(t))$ of system (2) is said to be stochastically ultimately bounded, if for any $\epsilon \in(0,1)$, there is a positive constant $\delta=\delta(\epsilon)$, such that, for any initial value $(x(\theta), y(\theta)) \in C\left([-\bar{\tau}, 0], R_{+}^{2}\right)$, the solution $X(t)$ to (2) has the property

$$
\limsup _{t \rightarrow+\infty} P\left(|X(t)|=\sqrt{x^{2}(t)+y^{2}(t)}>\delta\right)<\epsilon .
$$

Lemma 3.2 Let $\theta \in(0,1)$. Then there exists a positive constant $K=K(\theta)$, which is independent of the initial value $\left(\phi_{1}(\theta), \phi_{2}(\theta)\right) \in C\left([-\bar{\tau}, 0], R_{+}^{2}\right)$, such that the solution $X=(x, y)$ of system (2) has

$$
\lim \sup E|X|^{\theta} \leq K
$$

Proof Define $V(x, y)=x^{\theta}+y^{\theta}$. Applying Itô's formula to system (2), we obtain

$$
\begin{aligned}
d V(x, y)= & L V(x, y) d t+\sigma_{11} \theta x^{\theta} d B_{11}(t)+\sigma_{12} \theta x^{\theta+1} d B_{12}(t)+\sigma_{13} \theta x^{\theta} x\left(t-\tau_{1}(t)\right) d B_{13}(t) \\
& +\sigma_{14} \theta x^{\theta} y\left(t-\tau_{2}(t)\right) d B_{14}(t)+\sigma_{21} \theta y^{\theta} d B_{21}(t)+\sigma_{23} \theta y^{\theta+1} d B_{23}(t) \\
& +\sigma_{22} \theta y^{\theta} x\left(t-\tau_{3}(t)\right) d B_{22}(t)+\sigma_{24} \theta y^{\theta} y\left(t-\tau_{4}(t)\right) d B_{24}(t)
\end{aligned}
$$


where

$$
\begin{aligned}
L V(x, y)= & \theta x^{\theta}\left[r-a_{11} x-a_{12} x\left(t-\tau_{1}(t)\right)-a_{13} y\left(t-\tau_{2}(t)\right)\right]+\frac{\theta(\theta-1) \sigma_{11}^{2}}{2} x^{\theta} \\
& +\frac{\theta(\theta-1) \sigma_{12}^{2}}{2} x^{\theta+2}+\frac{\theta(\theta-1) \sigma_{13}^{2}}{2} x^{\theta} x^{2}\left(t-\tau_{1}(t)\right)+\frac{\theta(\theta-1) \sigma_{14}^{2}}{2} x^{\theta} y^{2}\left(t-\tau_{2}(t)\right) \\
& +\theta y^{\theta}\left[-d+a_{21} x\left(t-\tau_{3}(t)\right)-a_{22} y(t)-a_{23} y\left(t-\tau_{4}(t)\right)\right] d t+\frac{\theta(\theta-1) \sigma_{21}^{2}}{2} y^{\theta} \\
& +\frac{\theta(\theta-1) \sigma_{23}^{2}}{2} y^{\theta+2}+\frac{\theta(\theta-1) \sigma_{22}^{2}}{2} y^{\theta} x^{2}\left(t-\tau_{3}(t)\right)+\frac{\theta(\theta-1) \sigma_{24}^{2}}{2} y^{\theta} y^{2}\left(t-\tau_{4}(t)\right) \\
\leq & r \theta x^{\theta}+\theta a_{21} y^{\theta} x\left(t-\tau_{3}(t)\right)+\frac{\theta(\theta-1) \sigma_{12}^{2}}{2} x^{\theta+2}+\frac{\theta(\theta-1) \sigma_{23}^{2}}{2} y^{\theta+2} \\
\leq & r \theta x^{\theta}+\frac{\left(\theta a_{21}\right)^{2}}{4} y^{2 \theta}+\frac{\theta(\theta-1) \sigma_{12}^{2}}{2} x^{\theta+2}+\frac{\theta(\theta-1) \sigma_{23}^{2}}{2} y^{\theta+2}+x^{2}\left(t-\tau_{3}(t)\right) \\
= & F(x, y)-V(x, y)+x^{2}\left(t-\tau_{3}(t)\right)-\frac{e^{\bar{\tau}}}{1-\tau^{\prime}} x^{2}(t),
\end{aligned}
$$

where $F(x, y)=r \theta x^{\theta}+\frac{\left(\theta a_{21}\right)^{2}}{4} y^{2 \theta}+\frac{\theta(\theta-1) \sigma_{12}^{2}}{2} x^{\theta+2}+\frac{\theta(\theta-1) \sigma_{23}^{2}}{2} y^{\theta+2}+\frac{1-\tau^{\prime}+e^{\bar{\tau}}}{1-\tau^{\prime}} x^{2}+y^{2}$. Since $\theta \in(0,1)$, $2 \theta<\theta+2$, hence we easily see that $F(x, y)$ is bounded in $R_{+}^{2}$, namely,

$$
F(x, y) \leq M, \quad \forall X \in R_{+}^{2} .
$$

Hence, we have

$$
\begin{aligned}
d V(x, y) \leq & {\left[M-V(x, y)+x^{2}\left(t-\tau_{3}(t)\right)-\frac{e^{\bar{\tau}}}{1-\tau^{\prime}} x^{2}(t)\right] d t } \\
& +\sigma_{11} \theta x^{\theta} d B_{11}(t)+\sigma_{12} \theta x^{\theta+1} d B_{12}(t)+\sigma_{13} \theta x^{\theta} x\left(t-\tau_{1}(t)\right) d B_{13}(t) \\
& +\sigma_{14} \theta x^{\theta} y\left(t-\tau_{2}(t)\right) d B_{14}(t)+\sigma_{21} \theta y^{\theta} d B_{21}(t)+\sigma_{23} \theta y^{\theta+1} d B_{23}(t) \\
& +\sigma_{22} \theta y^{\theta} x\left(t-\tau_{3}(t)\right) d B_{22}(t)+\sigma_{24} \theta y^{\theta} y\left(t-\tau_{4}(t)\right) d B_{24}(t) .
\end{aligned}
$$

By virtue of (5), we use Itô's formula again for $e^{t} V(x, y)$. We have

$$
\begin{aligned}
d e^{t} V(x, y)= & e^{t}[V(x, y) d t+d V(x, y)] \\
\leq & e^{t}\left[M+x^{2}\left(t-\tau_{3}(t)\right)-\frac{e^{\bar{\tau}}}{1-\tau^{\prime}} x^{2}(t)\right] d t \\
& +\sigma_{11} \theta e^{t} x^{\theta} d B_{11}(t)+\sigma_{12} \theta e^{t} x^{\theta+1} d B_{12}(t)+\sigma_{13} \theta e^{t} x^{\theta} x\left(t-\tau_{1}(t)\right) d B_{13}(t) \\
& +\sigma_{14} \theta x^{\theta} e^{t} y\left(t-\tau_{2}(t)\right) d B_{14}(t)+\sigma_{21} \theta e^{t} y^{\theta} d B_{21}(t)+\sigma_{23} \theta e^{t} y^{\theta+1} d B_{23}(t) \\
& +\sigma_{22} \theta y^{\theta} e^{t} x\left(t-\tau_{3}(t)\right) d B_{22}(t)+\sigma_{24} \theta y^{\theta} e^{t} y\left(t-\tau_{4}(t)\right) d B_{24}(t),
\end{aligned}
$$

then

$$
\begin{aligned}
e^{t} E V(x, y) & \leq V(x(0), y(0))+M e^{t}-E \int_{0}^{t} \frac{e^{\bar{\tau}}}{1-\tau^{\prime}} e^{s} x^{2}(s) d s+E \int_{0}^{t} e^{s} x^{2}\left(s-\tau_{3}(s)\right) d s \\
& \leq V(x(0), y(0))+M e^{t}-E \int_{0}^{t} \frac{e^{\bar{\tau}}}{1-\tau^{\prime}} e^{s} x^{2}(s) d s
\end{aligned}
$$




$$
\begin{aligned}
& +E\left[\frac{e^{\bar{\tau}}}{1-\tau^{\prime}} \int_{0}^{t} e^{\left(s-\tau_{3}(s)\right)} x^{2}\left(s-\tau_{3}(s)\right) d\left(s-\tau_{3}(s)\right)\right] \\
\leq & V(x(0), y(0))+M e^{t}+\frac{e^{\bar{\tau}}}{1-\tau^{\prime}} \int_{-\tau_{3}(0)}^{0} e^{s} x^{2}(s) d s .
\end{aligned}
$$

We easily infer that

$$
\limsup _{t \rightarrow+\infty} E V(x, y) \leq M
$$

On the other hand,

$$
|X|^{\theta}=\left(x^{2}+y^{2}\right)^{\frac{\theta}{2}} \leq 2^{\frac{\theta}{2}} \max \left(x^{\theta}, y^{\theta}\right) \leq 2^{\frac{\theta}{2}} V(x, y) .
$$

Hence,

$$
\limsup _{t \rightarrow+\infty} E|X|^{\theta} \leq 2^{\frac{\theta}{2}} M:=K
$$

The proof is completed.

Theorem 3.3 Let $\theta \in(0,1)$, and system (2) is stochastically ultimate bounded.

Proof From Lemma 3.2, we know there is a positive constant $K$, such that $\lim \sup _{t \rightarrow+\infty} E|X|^{\theta} \leq K$. For any $\epsilon>0$, let $\delta=K^{2} / \epsilon^{2}$. By Chebyshev's inequality, we have

$$
P\{|X(t)|>\delta\} \leq \frac{\sqrt{|X(t)|}}{\sqrt{\delta}}
$$

Then

$$
\limsup _{t \rightarrow+\infty} P\{|X(t)|>\delta\} \leq \epsilon
$$

\section{Asymptotic pathwise estimation}

Lemma 2.1 and Theorem 3.3 show that the global positive solution of system (2) is stochastically ultimate bounded. This nice property motivates us to further study the system. In this section, we will study the pathwise properties of system (2).

Theorem 4.1 For any given initial value satisfying $\left(\phi_{1}(\theta), \phi_{2}(\theta)\right) \in C\left([-\bar{\tau}, 0], R_{+}^{2}\right)$, the solutions $(x(t), y(t))$ of system (2) have the properties

$$
\limsup _{t \rightarrow+\infty} \frac{\ln x(t)}{\ln t} \leq 1, \quad \limsup _{t \rightarrow+\infty} \frac{\ln y(t)}{\ln t} \leq 1 .
$$

Proof Applying Itô's formula to system (2), we have

$$
\begin{aligned}
d e^{t} \ln V(x, y) & =e^{t} \ln (x+y) d t+e^{t} d \ln (x+y) \\
& =e^{t} \ln (x+y)+\frac{e^{t} x}{x+y}\left[r-a_{11} x-a_{12} x\left(t-\tau_{1}(t)\right)-a_{13} y\left(t-\tau_{2}(t)\right)\right] d t
\end{aligned}
$$




$$
\begin{aligned}
& -0.5 \frac{e^{t} x^{2}}{(x+y)^{2}}\left[\sigma_{11}^{2}+\sigma_{12}^{2} x^{2}+\sigma_{13}^{2} x^{2}\left(t-\tau_{1}(t)\right)+\sigma_{14}^{2} y^{2}\left(t-\tau_{2}(t)\right)\right] d t \\
& +\frac{e^{t} x}{x+y}\left[\sigma_{11} d B_{11}(t)+\sigma_{12} x d B_{12}(t)+\sigma_{13} x\left(t-\tau_{1}(t)\right) d B_{13}(t)\right. \\
& \left.+\sigma_{14} y\left(t-\tau_{2}(t)\right) d B_{14}(t)\right] \\
& +\frac{e^{t} y}{x+y}\left[-d+a_{21} x\left(t-\tau_{3}(t)\right)-a_{22} y(t)-a_{23} y\left(t-\tau_{4}(t)\right)\right] d t \\
& -0.5 \frac{e^{t} y^{2}}{(x+y)^{2}}\left[\sigma_{21}^{2}+\sigma_{23}^{2} y^{2}+\sigma_{22}^{2} x^{2}\left(t-\tau_{3}(t)\right)+\sigma_{24}^{2} y^{2}\left(t-\tau_{4}(t)\right)\right] d t \\
& +\frac{e^{t} y}{x+y}\left[\sigma_{21} d B_{21}(t)+\sigma_{23} y d B_{23}(t)+\sigma_{22} x\left(t-\tau_{3}(t)\right) d B_{22}(t)\right. \\
& \left.+\sigma_{24} y\left(t-\tau_{4}(t)\right) d B_{24}(t)\right],
\end{aligned}
$$

thus

$$
\begin{aligned}
e^{t} \ln (x(t)+y(t))= & \ln (x(0)+y(0))+\int_{0}^{t} e^{s} \ln (x(s)+y(s)) d s \\
& +\int_{0}^{t} \frac{e^{s} x(s)}{x(s)+y(s)}\left[r-a_{11} x(s)-a_{12} x\left(s-\tau_{1}(s)\right)-a_{13} y\left(s-\tau_{2}(s)\right)\right] d s \\
& -0.5 \int_{0}^{t} \frac{e^{s} x^{2}(s)}{(x(s)+y(s))^{2}}\left[\sigma_{11}^{2}+\sigma_{12}^{2} x^{2}(s)+\sigma_{13}^{2} x^{2}\left(s-\tau_{1}(s)\right)\right. \\
& \left.+\sigma_{14}^{2} y^{2}\left(s-\tau_{2}(s)\right)\right] d s \\
& +\int_{0}^{t} \frac{e^{s} y(s)}{x(s)+y(s)}\left[-d+a_{21} x\left(s-\tau_{3}(s)\right)-a_{22} y(s)-a_{23} y\left(s-\tau_{4}(s)\right)\right] d s \\
& -\int_{0}^{t} 0.5 \frac{e^{s} y^{2}(s)}{(x(s)+y(s))^{2}}\left[\sigma_{21}^{2}+\sigma_{23}^{2} y^{2}(s)+\sigma_{22}^{2} x^{2}\left(s-\tau_{3}(s)\right)\right. \\
& \left.+\sigma_{24}^{2} y^{2}\left(s-\tau_{4}(s)\right)\right] d s \\
& +M_{1}(t)+M_{2}(t)+M_{3}(t)+M_{4}(t) \\
& +M_{5}(t)+M_{6}(t)+M_{7}(t)+M_{8}(t)
\end{aligned}
$$

where

$$
\begin{array}{ll}
M_{1}(t)=\int_{0}^{t} \sigma_{11} \frac{e^{s} x(s)}{x(s)+y(s)} d B_{11}(s), & M_{2}(t)=\int_{0}^{t} \sigma_{12} \frac{e^{s} x^{2}(s)}{x(s)+y(s)} d B_{12}(s), \\
M_{3}(t)=\int_{0}^{t} \sigma_{13} \frac{e^{s} x(s) x\left(s-\tau_{1}(s)\right)}{x(s)+y(s)} d B_{13}(s), & M_{4}(t)=\int_{0}^{t} \sigma_{14} \frac{e^{s} x(s) y\left(s-\tau_{2}(s)\right)}{x(s)+y(s)} d B_{14}(s), \\
M_{5}(t)=\int_{0}^{t} \sigma_{21} \frac{e^{s} y(s)}{x(s)+y(s)} d B_{21}(s), & M_{6}(t)=\int_{0}^{t} \sigma_{22} \frac{e^{s} y(s) x\left(s-\tau_{3}(s)\right)}{x(s)+y(s)} d B_{22}(s), \\
M_{7}(t)=\int_{0}^{t} \sigma_{23} \frac{e^{s} y^{2}(s)}{x(s)+y(s)} d B_{23}(s), & M_{8}(t)=\int_{0}^{t} \sigma_{24} \frac{e^{s} y(s) y\left(s-\tau_{4}(s)\right)}{x(s)+y(s)} d B_{24}(s) .
\end{array}
$$


Let $M(t)=\sum_{i=1}^{8} M_{i}(t)$. Then $M(t)$ is a local martingale, and the quadratic form of $M(t)$ is

$$
\begin{aligned}
\langle M(t), M(t)\rangle= & 0.5 \int_{0}^{t} \frac{e^{2 s} x^{2}(s)}{(x(s)+y(s))^{2}}\left[\sigma_{11}^{2}+\sigma_{12}^{2} x^{2}(s)+\sigma_{13}^{2} x^{2}\left(s-\tau_{1}(s)\right)\right. \\
& \left.+\sigma_{14}^{2} y^{2}\left(s-\tau_{2}(s)\right)\right] d s \\
& +0.5 \int_{0}^{t} \frac{e^{2 s} y^{2}(s)}{(x(s)+y(s))^{2}}\left[\sigma_{21}^{2}+\sigma_{23}^{2} y^{2}(s)+\sigma_{22}^{2} x^{2}\left(s-\tau_{3}(s)\right)\right. \\
& \left.+\sigma_{24}^{2} y^{2}\left(s-\tau_{4}(s)\right)\right] d s .
\end{aligned}
$$

According to the exponential martingale inequality (see [17]), for any positive constants $T, \alpha$, and $\beta$ we have

$$
P\left\{\sup _{0 \leq t \leq T}\left[M(t)-\frac{\alpha}{2}\langle M(t), M(t)\rangle\right]>\beta\right\} \leq e^{-\alpha \beta} .
$$

We choose $T=\gamma k, \alpha=e^{-\gamma k}, \beta=\rho e^{\gamma k} \ln k$, and then

$$
P\left\{\sup _{0 \leq t \leq \gamma k}\left[M(t)-\frac{e^{-\gamma k}}{2}\langle M(t), M(t)\rangle\right]>\rho e^{\gamma k} \ln k\right\} \leq k^{-\rho},
$$

where $\rho>1$ and $\gamma>0$. By virtue of the Borel-Cantelli lemma [13], for almost all $\omega \in \Omega$, there exists a $k_{0}(\omega)$, such that, for all $k \geq k_{0}(\omega)$,

$$
M(t) \leq \frac{e^{-\gamma k}}{2}\langle M(t), M(t)\rangle+\rho e^{\gamma k} \ln k, \quad 0 \leq t \leq \gamma k .
$$

Substituting the above inequality into equation (6), we have

$$
\begin{aligned}
e^{t} \ln (x(t)+y(t)) \leq & \ln (x(0)+y(0))+\int_{0}^{t} e^{s} \ln (x(s)+y(s)) d s+\rho e^{\gamma k} \ln k \\
& +\int_{0}^{t} \frac{e^{s} x(s)}{x(s)+y(s)}\left[r-a_{11} x(s)\right] d s \\
& +\int_{0}^{t} \frac{e^{s} y(s)}{x(s)+y(s)}\left[a_{21} x\left(s-\tau_{3}(s)\right)-a_{22} y(s)\right] d s \\
& -0.5 \int_{0}^{t} \frac{e^{s}\left(1-e^{s-\gamma k}\right) x^{2}(s)}{(x(s)+y(s))^{2}}\left[\sigma_{11}^{2}+\sigma_{12}^{2} x^{2}(s)+\sigma_{13}^{2} x^{2}\left(s-\tau_{1}(s)\right)\right. \\
& \left.+\sigma_{14}^{2} y^{2}\left(s-\tau_{2}(s)\right)\right] d s \\
& -0.5 \int_{0}^{t} \frac{e^{s}\left(1-e^{s-\gamma k}\right) y^{2}(s)}{(x(s)+y(s))^{2}}\left[\sigma_{21}^{2}+\sigma_{23}^{2} y^{2}(s)+\sigma_{22}^{2} x^{2}\left(s-\tau_{3}(s)\right)\right. \\
& \left.+\sigma_{24}^{2} y^{2}\left(s-\tau_{4}(s)\right)\right] d s .
\end{aligned}
$$

Because $0 \leq t \leq \gamma k, k \geq K_{0}(\omega)$, we know $s \leq \gamma k$. Note that

$$
\begin{aligned}
\int_{0}^{t} e^{s} x\left(s-\tau_{3}(s)\right) d s & \leq \int_{0}^{t} \frac{e^{s-\tau_{3}(s)} e^{\tau_{3}(s)} x\left(s-\tau_{3}(s)\right)}{1-\tau^{\prime}} d\left(s-\tau_{3}(s)\right) \leq \frac{e^{\bar{\tau}}}{1-\tau^{\prime}} \int_{-\tau_{3}(0)}^{t-\tau_{3}(t)} e^{s} x(s) d s \\
& \leq \frac{e^{\bar{\tau}}}{1-\tau^{\prime}} \int_{-\tau_{3}(0)}^{0} e^{s} x(s) d s+\frac{e^{\bar{\tau}}}{1-\tau^{\prime}} \int_{0}^{t} e^{s} x(s) d s .
\end{aligned}
$$


Then we can rewrite (8)

$$
\begin{aligned}
e^{t} \ln (x(t)+y(t)) \leq & \ln (x(0)+y(0))+\frac{a_{21} e^{\bar{\tau}}}{1-\tau^{\prime}} \int_{-\tau_{3}(0)}^{0} e^{s} x(s) d s \\
& +\int_{0}^{t} e^{s}[\ln (x(s)+y(s))+r] d s+\frac{a_{21} e^{\bar{\tau}}}{1-\tau^{\prime}} \int_{0}^{t} e^{s} x(s) d s \\
& -\int_{0}^{t} \frac{\min \left\{\sigma_{12}^{2}, \sigma_{23}^{2}\right\} e^{s}\left(1-e^{s-\gamma k}\right)\left(x^{4}(s)+y^{4}(s)\right)}{2(x(s)+y(s))^{2}} d s+\rho e^{\gamma k} \ln k \\
\leq & \ln (x(0)+y(0))+\frac{a_{21} e^{\bar{\tau}}}{1-\tau^{\prime}} \int_{-\tau_{3}(0)}^{0} e^{s} x(s) d s+\rho e^{\gamma k} \ln k \\
& +\int_{0}^{t} e^{s}\left[\ln (x+y)+r+\frac{a_{21} e^{\bar{\tau}}}{1-\tau^{\prime}}[x+y]\right. \\
& \left.-\frac{\min \left\{\sigma_{12}^{2}, \sigma_{23}^{2}\right\}\left(1-e^{s-\gamma k}\right)}{16}(x+y)^{2}\right] d s .
\end{aligned}
$$

Obviously, there is a positive constant $K_{1}$ such that

$$
\ln (x+y)+r+\frac{a_{21} e^{\bar{\tau}}}{1-\tau^{\prime}}[x+y]-\frac{\min \left\{\sigma_{12}^{2}, \sigma_{23}^{2}\right\}\left(1-e^{t-\gamma k}\right)}{16}(x+y)^{2} \leq K_{1} .
$$

Let $C=\ln (x(0)+y(0))+\frac{a_{21} e^{\bar{\tau}}}{1-\tau^{\prime}} \int_{-\tau_{3}(0)}^{0} e^{s} x(s) d s$. Then, for all $0 \leq t \leq \gamma k, k \geq K_{0}(\omega)$,

$$
e^{t} \ln (x(t)+y(t)) \leq C+\int_{0}^{t} K_{1} e^{s} d s+\rho e^{\gamma k} \ln k
$$

If $\gamma(k-1) \leq t \leq \gamma k$ and $k \geq k_{0}(\omega)$, we obtain

$$
\frac{\ln (x(t)+y(t))}{\ln t} \leq \frac{C}{e^{t} \ln t}+\frac{K_{1} e^{t}-K_{1}}{e^{t} \ln t}+\frac{\rho e^{\gamma k} \ln k}{e^{t} \ln t} .
$$

We infer

$$
\limsup _{t \rightarrow+\infty} \frac{\ln (x(t)+y(t))}{\ln t} \leq \rho e^{\gamma}
$$

Letting $\rho \rightarrow 1, \gamma \rightarrow 0$, by (10) we have

$$
\limsup _{t \rightarrow+\infty} \frac{\ln (x(t))}{\ln t} \leq 1, \quad \limsup _{t \rightarrow+\infty} \frac{\ln (y(t))}{\ln t} \leq 1, \quad \text { a.s. }
$$

\section{Persistence and extinction}

It is critical to discuss the persistence and extinction for an ecological population system. In this section, we will mainly investigate the persistence in mean and extinction of system (2).

\section{Theorem 5.1}

(1) If $r<0.5 \sigma_{11}^{2}$, populations $x$ and $y$ of system (2) will go extinct a.s., namely, $\lim _{t \rightarrow+\infty} x(t)=\lim _{t \rightarrow+\infty} y(t)=0$, a.s. 
(2) If $r>0.5 \sigma_{11}^{2}$, population $x$ is weakly persistent a.s., namely, $x^{*}>0$.

(a) If $r>0.5 \sigma_{11}^{2}$ and $\frac{a_{21}\left(r-0.5 \sigma_{11}^{2}\right)}{\left(1-\tau^{\prime}\right) a_{11}}-\left(d+0.5 \sigma_{21}^{2}\right)<0$, population $y$ of system (2) will go extinct a.s., namely, $\lim _{t \rightarrow+\infty} y(t)=0$, a.s.

(b) If $r>0.5 \sigma_{11}^{2}$ and $\frac{a_{21}\left(r-0.5 \sigma_{11}^{2}\right)}{\left(1-\tau^{\prime}\right) a_{11}}-\left(d+0.5 \sigma_{21}^{2}\right)>0$, then $\langle y(t)\rangle^{*} \leq \frac{\frac{a_{21}\left(r-0.5 \sigma_{11}^{2}\right)}{\left(1-\tau^{\prime}\right) a_{11}}-\left(d+0.5 \sigma_{21}^{2}\right)}{a_{22}}$, a.s.

(3) If $r=0.5 \sigma_{11}^{2}$, then the population $x$ is nonpersistent in the mean a.s., namely, $\langle x\rangle^{*}=0$.

Proof Applying Itô's formula to system (2), we have

$$
\begin{aligned}
d \ln x= & {\left[r-a_{11} x-a_{12} x\left(t-\tau_{1}(t)\right)-a_{13} y\left(t-\tau_{2}(t)\right)\right] d t } \\
& -0.5\left[\sigma_{11}^{2}+\sigma_{12}^{2} x^{2}+\sigma_{13}^{2} x^{2}\left(t-\tau_{1}(t)\right)+\sigma_{14}^{2} y^{2}\left(t-\tau_{2}(t)\right)\right] d t \\
& +\sigma_{11} d B_{11}(t)+\sigma_{12} x d B_{12}(t)+\sigma_{13} x\left(t-\tau_{1}(t)\right) d B_{13}(t) \\
& +\sigma_{14} y\left(t-\tau_{2}(t)\right) d B_{14}(t), \\
d \ln y=[ & \left.-d+a_{21} x\left(t-\tau_{3}(t)\right)-a_{22} y(t)-a_{23} y\left(t-\tau_{4}(t)\right)\right] d t \\
& -0.5\left[\sigma_{21}^{2}+\sigma_{23}^{2} y^{2}+\sigma_{22}^{2} x^{2}\left(t-\tau_{3}(t)\right)+\sigma_{24}^{2} y^{2}\left(t-\tau_{4}(t)\right)\right] d t \\
& +\sigma_{21} d B_{21}(t)+\sigma_{23} y d B_{23}(t)+\sigma_{22} x\left(t-\tau_{3}(t)\right) d B_{22}(t) \\
& +\sigma_{24} y\left(t-\tau_{4}(t)\right) d B_{24}(t) .
\end{aligned}
$$

Integrating both sides from 0 to $t$, we get

$$
\begin{aligned}
\ln x(t) / x(0)= & \int_{0}^{t} r-a_{11} x(s)-a_{12} x\left(s-\tau_{1}(s)\right)-a_{13} y\left(s-\tau_{2}(s)\right) d s \\
& -0.5 \int_{0}^{t} \sigma_{11}^{2}+\sigma_{12}^{2} x^{2}(s)+\sigma_{13}^{2} x^{2}\left(s-\tau_{1}(s)\right)+\sigma_{14}^{2} y^{2}\left(s-\tau_{2}(s)\right) d s \\
& +\int_{0}^{t} \sigma_{11} d B_{11}(s)+\int_{0}^{t} \sigma_{12} x(s) d B_{12}(s)+\int_{0}^{t} \sigma_{13} x\left(s-\tau_{1}(s)\right) d B_{13}(s) \\
& +\int_{0}^{t} \sigma_{14} y\left(s-\tau_{2}(s)\right) d B_{14}(s), \\
\ln y(t) / y(0)= & \int_{0}^{t}-d+a_{21} x\left(s-\tau_{3}(s)\right)-a_{22} y(s)-a_{23} y\left(s-\tau_{4}(s)\right) d s \\
& -0.5 \int_{0}^{t} \sigma_{21}^{2}+\sigma_{23}^{2} y^{2}(s)+\sigma_{22}^{2} x^{2}\left(s-\tau_{3}(s)\right)+\sigma_{24}^{2} y^{2}\left(s-\tau_{4}(s)\right) d s \\
& +\int_{0}^{t} \sigma_{21} d B_{21}(s)+\int_{0}^{t} \sigma_{23} y(s) d B_{23}(s)+\int_{0}^{t} \sigma_{22} x\left(s-\tau_{3}(s)\right) d B_{22}(s) \\
& +\int_{0}^{t} \sigma_{24} y\left(s-\tau_{4}(s)\right) d B_{24}(s),
\end{aligned}
$$

where

$$
\begin{aligned}
& N_{1}(t)=\int_{0}^{t} \sigma_{11} d B_{11}(s), \quad N_{2}(t)=\int_{0}^{t} \sigma_{12} x(s) d B_{12}(s), \\
& N_{3}(t)=\int_{0}^{t} \sigma_{13} x\left(s-\tau_{1}(s)\right) d B_{13}(s), \quad N_{4}(t)=\int_{0}^{t} \sigma_{14} y\left(s-\tau_{2}(s)\right) d B_{14}(s),
\end{aligned}
$$




$$
\begin{aligned}
& N_{5}(t)=\int_{0}^{t} \sigma_{21} d B_{21}(s), \quad N_{6}(t)=\int_{0}^{t} \sigma_{22} x\left(s-\tau_{3}(s)\right) d B_{22}(s), \\
& N_{7}(t)=\int_{0}^{t} \sigma_{23} y(s) d B_{23}(s), \quad N_{8}(t)=\int_{0}^{t} \sigma_{24} y\left(s-\tau_{4}(s)\right) d B_{24}(s) .
\end{aligned}
$$

Noting that $N_{i}(t)$ is a local martingale, we have

$$
\begin{aligned}
& \left\langle N_{2}(t), N_{2}(t)\right\rangle=\int_{0}^{t} \sigma_{21}^{2} x^{2}(s) d s, \quad\left\langle N_{3}(t), N_{3}(t)\right\rangle=\int_{0}^{t} \sigma_{23}^{2} x^{2}\left(s-\tau_{1}(s)\right) d s, \\
& \left\langle N_{4}(t), N_{4}(t)\right\rangle=\int_{0}^{t} \sigma_{14}^{2} y^{2}\left(s-\tau_{2}(s)\right) d s, \quad\left\langle N_{6}(t), N_{6}(t)\right\rangle=\int_{0}^{t} \sigma_{22}^{2} x^{2}\left(s-\tau_{3}(s)\right) d s, \\
& \left\langle N_{7}(t), N_{7}(t)\right\rangle=\int_{0}^{t} \sigma_{23}^{2} y^{2}(s) d s, \quad\left\langle N_{8}(t), N_{8}(t)\right\rangle=\int_{0}^{t} \sigma_{24}^{2} y^{2}\left(s-\tau_{4}(s)\right) d s .
\end{aligned}
$$

By the exponential martingale inequality (7) (choose $T=k, \alpha=1, \beta=2 \ln k$ ), we have

$$
P\left\{\sup _{0 \leq t \leq \gamma k}\left[N_{i}(t)-\frac{1}{2}\left\langle N_{i}(t), N_{i}(t)\right\rangle\right]>2 \ln k\right\} \leq 1 / k^{2}, \quad i=2,3,4,6,7,8 .
$$

By virtue of the Borel-Cantelli lemma, for almost all $\omega \in \Omega$, there exists a $k_{0}(\omega)$ such that, for all $k \geq k_{0}(\omega)$,

$$
N_{i}(t) \leq \frac{1}{2}\left\langle N_{i}(t), N_{i}(t)\right\rangle+2 \ln k, \quad 0 \leq t \leq k, k \geq k_{0}(\omega) .
$$

Substituting above inequalities into (11) and (12), we have

$$
\begin{aligned}
\ln x(t) / x(0) \leq & \int_{0}^{t} r-0.5 \sigma_{11}^{2}-a_{11} x(s)-a_{12} x\left(s-\tau_{1}(s)\right)-a_{13} y\left(s-\tau_{2}(s)\right) d s \\
& +N_{1}(t)+6 \ln k \\
\leq & \int_{0}^{t} r-0.5 \sigma_{11}^{2} d s+N_{1}(t)+6 \ln k, \\
\ln y(t) / y(0) \leq & \int_{0}^{t}-d-0.5 \sigma_{21}^{2}+a_{21} x\left(s-\tau_{3}(s)\right)-a_{22} y(s)-a_{23} y\left(s-\tau_{4}(s)\right) d s \\
& +N_{5}(t)+6 \ln k \\
\leq & \int_{0}^{t}-d-0.5 \sigma_{21}^{2}+a_{21} x\left(s-\tau_{3}(s)\right) d s+N_{5}(t)+6 \ln k,
\end{aligned}
$$

for all $0 \leq t \leq k, k \geq k_{0}$. When $0 \leq k-1 \leq t \leq k$, by (13)

$$
\frac{\ln x(t) / x(0)}{t} \leq r-0.5 \sigma_{11}^{2}+\frac{N_{1}(t)}{t}+\frac{6 \ln k}{k-1}
$$

(1) If $r-0.5 \sigma_{11}^{2}<0$, have $\lim _{t \rightarrow+\infty} x(t)=0$. In combination with (14) we easily obtain $\lim _{t \rightarrow+\infty} y(t)=0$ a.s. 
(2) If $r-0.5 \sigma_{11}^{2}>0$, suppose this assertion is not true, that is to say $P(S)>0$, where $S=\left\{x^{*}=0\right\}$. Then for $\omega \in S$, we have $\lim _{t \rightarrow+\infty} x(t, \omega)=0$, hence,

$$
\begin{aligned}
\lim _{t \rightarrow+\infty}\langle x(t, \omega)\rangle & =\lim _{t \rightarrow+\infty}\left\langle x^{2}(t, \omega)\right\rangle=\lim _{t \rightarrow+\infty}\left\langle x\left(t-\tau_{1}(t), \omega\right)\right\rangle=\lim _{t \rightarrow+\infty}\left\langle x^{2}\left(t-\tau_{1}(t), \omega\right)\right\rangle \\
& =\lim _{t \rightarrow+\infty}\left\langle y\left(t-\tau_{2}(t), \omega\right)\right\rangle=\lim _{t \rightarrow+\infty}\left\langle y^{2}\left(t-\tau_{2}(t), \omega\right)\right\rangle=0 .
\end{aligned}
$$

Then, according to the law of large numbers for local martingales, $\frac{N_{i}(t)}{t}=0$. By virtue of (12) and (15) we have

$$
\left[\frac{\ln x(t) / x(0)}{t}\right]^{*}=r-0.5 \sigma_{11}^{2}>0
$$

By Theorem 4.1 we obtain

$$
\left[\frac{\ln x(t) / x(0)}{t}\right]^{*} \leq\left[\frac{\ln x(t) / x(0)}{\ln t}\right]^{*}\left[\frac{\ln t}{t}\right]^{*} \leq 0
$$

Obviously, this is a contradiction, namely $x^{*}>0$.

For all $0 \leq t \leq k, k \geq k_{0}$. Then, $\forall \epsilon>0$, there exists a $T>0$, when $0<k-1 \leq T \leq t \leq k$ and $\left[\frac{6 \ln k}{t}\right]^{*}<\epsilon$.

It follows from (13) that

$$
\frac{\ln x(t) / x(0)}{t} \leq r-0.5 \sigma_{11}^{2}+\epsilon-a_{11}|x(t)\rangle+\frac{N_{1}(t)}{t}, \quad t \geq T .
$$

If $r-0.5 \sigma_{11}^{2}>0$, we have $\langle x(t)\rangle^{*} \leq \frac{r-0.5 \sigma_{11}^{2}}{a_{11}}$.

In the same way, by (14)

$$
\begin{aligned}
\frac{\ln y(t) / y(0)}{t} \leq & -d-0.5 \sigma_{21}^{2}+t^{-1} \int_{0}^{t} a_{21} x\left(s-\tau_{3}(s)\right) d s-a_{22}\langle y(t)\rangle+\frac{N_{5}(t)}{t}+\frac{6 \ln k}{t} \\
\leq & -d-0.5 \sigma_{21}^{2}+\frac{a_{21}}{t\left(1-\tau^{\prime}\right)}\left[\int_{-\tau(0)}^{0} x(s) d s+\int_{0}^{t} x(s) d s\right]-a_{22}\langle y(t)\rangle \\
& +\frac{N_{5}(t)}{t}+\frac{6 \ln k}{t}
\end{aligned}
$$

for all $0 \leq t \leq k, k \geq k_{0} . \forall \epsilon>0$, there exists a $T>0$, when $0<k-1 \leq T \leq t \leq k,\left[\frac{6 \ln k}{t}\right]^{*}<$ $\epsilon / 2$ and $\frac{a_{21}}{t\left(1-\tau^{\prime}\right)} \int_{-\tau(0)}^{0} x(s) d s<\epsilon / 2$.

We have

$$
\frac{\ln y(t) / y(0)}{t} \leq \frac{a_{21}\left(r-0.5 \sigma_{11}^{2}\right)}{a_{11}\left(1-\tau^{\prime}\right)}-\left(d+0.5 \sigma_{21}^{2}\right)-a_{22}\langle y(t)\rangle+\frac{N_{5}(t)}{t} .
$$

(a) If $\frac{a_{21}\left(r-0.5 \sigma_{11}^{2}\right)}{a_{11}\left(1-\tau^{\prime}\right)}-\left(d+0.5 \sigma_{21}^{2}\right)<0$, we have $\lim _{t \rightarrow+\infty} y(t)=0$, a.s. (b) If $\frac{a_{21}\left(r-0.5 \sigma_{11}^{2}\right)}{a_{11}\left(1-\tau^{\prime}\right)}-$ $\left(d+0.5 \sigma_{21}^{2}\right)>0$, we have $\langle y(t)\rangle^{*} \leq \frac{\frac{a_{21}\left(r-0.5 \sigma_{11}^{2}\right)}{\left(1-\tau^{\prime}\right) a_{11}}-\left(d+0.5 \sigma_{21}^{2}\right)}{a_{22}}$, a.s.

(3) If $r-0.5 \sigma_{11}^{2}=0$, by (17) we have $\langle x(t)\rangle^{*} \leq \frac{\epsilon}{a_{11}}$. Since $\epsilon$ is arbitrary, we have $\langle x(t)\rangle^{*}=0$, a.s.

The proof of Theorem 5.1 is completed. 


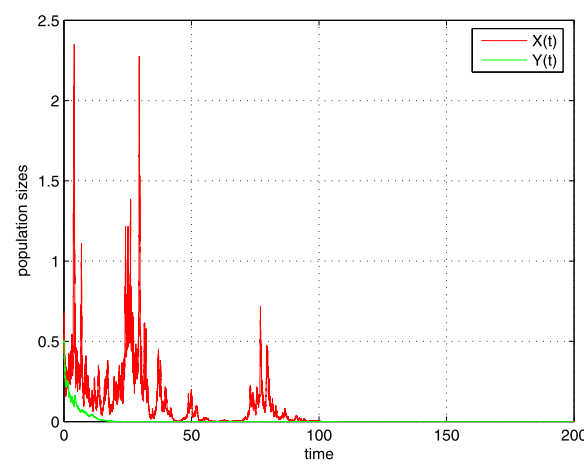

(a)

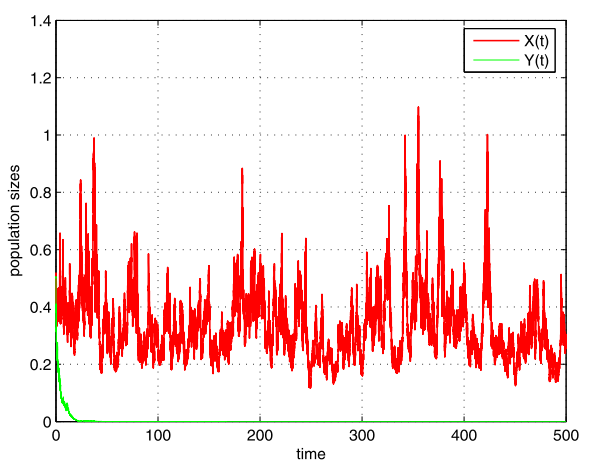

(b)

Figure 1 Solution of system (2) for $r=0.3, a_{11}=0.6, a_{12}=0.2, a_{13}=0.5, d=0.4, a_{21}=0.6, a_{22}=0.4$, $a_{23}=0.3, \tau_{1}(t)=0.2(2+\cos t), \tau_{2}(t)=0.3(1+\sin t), \tau_{3}(t)=0.2(2+\sin t), \tau_{4}(t)=0.3(1+\sin t), \sigma_{12}=0.2$, $\sigma_{13}=\mathbf{0 . 4}, \sigma_{14}=\mathbf{0 . 2}, \sigma_{21}=\sigma_{22}=\sigma_{23}=\sigma_{24}=\mathbf{0 . 2}$. (a) $\sigma_{11}=0.8, \sigma_{21}=0.2$. (b) $\sigma_{11}=0.2, \sigma_{21}=0.2$.

Remark 5.1 The definition of weak persistence is not a very appropriate one for stochastic models. Many authors have introduced some more appropriate definitions of permanence for stochastic population models, for example, stochastic persistence in probability (see $[21,22]$ ) or a new definition of stochastic permanence (see [23]). We will continue to study them in future work.

\section{Numerical simulations}

Now let us use Milstein's method (see [24]) to support our results. See Figure 1. We choose $r=0.3, a_{11}=0.6, a_{12}=0.2, a_{13}=0.5, d=0.4, a_{21}=0.6, a_{22}=0.4, a_{23}=0.3, \tau_{1}(t)=0.2 \times$ $(2+\cos t), \tau_{2}(t)=0.3(1+\sin t), \tau_{3}(t)=0.2(2+\sin t), \tau_{4}(t)=0.3(1+\sin t), \sigma_{12}=0.2, \sigma_{13}=0.4$, $\sigma_{14}=0.2, \sigma_{21}=\sigma_{22}=\sigma_{23}=\sigma_{24}=0.2$. The difference between the conditions of Figure 1(a) and Figure $1(\mathrm{~b})$ are the values of $\sigma_{11}$ and $\sigma_{21}$. In Figure $1(\mathrm{a})$, we choose $\sigma_{11}=0.8, \sigma_{21}=0.2$. Then $r-0.5 \sigma_{11}^{2}<0$, hence, by Theorem 5.1(1), we see that populations $x$ and $y$ go extinct; see Figure $1(\mathrm{a})$. In Figure $1(\mathrm{~b})$, we choose $\sigma_{11}=0.2, \sigma_{21}=0.2$. Then, by computation, we obtain $r-0.5 \sigma_{11}^{2}>0$ and $\frac{a_{21}\left(r-0.5 \sigma_{11}^{2}\right)}{a_{11}\left(1-\tau^{\prime}\right)}-\left(d+0.5 \sigma_{21}^{2}\right)<0$, hence, by Theorem 5.1 , we see that population $x$ is weakly persistent and population $y$ goes extinct; see Figure 1(b).

\section{Conclusions}

In this paper, we consider a stochastic predator-prey system with time-dependent delays. We first show that system (2) has a global positive solution and is stochastically ultimate bounded. And then we also discuss the asymptotic properties for the moments as well as the sample paths of the solution. Finally, we obtain the critical value between weak persistence and extinction of the prey and simulate the model to support our results.

There are still many interesting and challenging questions that need to be studied. We outline some open problems and topics for further research.

(1) The stability of the positive equilibrium state is one of the most interesting topics in the study of population models. Considering the influence of environment noise, the stochastic models do not keep the positive equilibrium state of the corresponding deterministic systems. However, in recent years, many authors have studied the stability in distribution of stochastic population models (see [25-27]). Hence, the 
study about the stability in distribution of system (2) is necessary. We will continue to investigate this in our future work.

(2) In the natural environment, the populations are inevitably subject to the environmental noises, and when the intensity of the noise is sufficiently large, the population will go extinct (see Figure 1(a)). Hence, we can extend classical deterministic systems with time dependence. For example, in $[9,10]$, the authors considered a population model:

$$
\dot{x}(t)=\sum_{k=1}^{m} \alpha_{k}(t) x\left(h_{k}(t)\right)-\beta(t) x^{2}(t), \quad t \geq 0,
$$

where $m \geq 1$ is an integer, and functions $h_{k}:[0, \infty) \rightarrow R$ are continuous, such that $t-\tau \leq h_{k}(t) \leq t, \tau=$ constant. They considered the effects of environmental noise, and studied the stochastic permanence, the stability in distribution and other dynamic properties of stochastic population models.

\begin{abstract}
Acknowledgements
This paper is supported by the Cooperation Agreement Project by the Department of Science and Technology of Guizhou Province of China (LH[2016]7300), and the Project for Innovative Research Groups of Guizhou Province of China (KY[2016]051)
\end{abstract}

\title{
Competing interests
}

The authors declare that they have no competing interests.

\section{Authors' contributions}

All authors contributed equally to the writing of this paper. The authors read and approved the final manuscript.

\section{Publisher's Note}

Springer Nature remains neutral with regard to jurisdictional claims in published maps and institutional affiliations.

Received: 22 March 2017 Accepted: 12 August 2017 Published online: 25 September 2017

\section{References}

1. Freedman, HI: Deterministic Mathematical Models in Population Ecology. Marcel Dekker, New York (1980)

2. Freedman, HI, Waltman, P: Persistence in models of three interacting predator-prey populations. Math. Biosci. 68 , 213-231 (1984)

3. Teng, ZD, Yu, YH: The extinction in nonautonomous prey-predator Lotka-Volterra systems. Acta Math. Appl. Sin. 15(4), 401-408 (1999)

4. Kuang, Y: Delay Differential Equations with Applications in Population Dynamics. Academic Press, Boston (1993)

5. Pao, C: Global asymptotic stability of Lotka-Volterra 3-species reaction-diffusion systems with time delays. J. Math. Anal. Appl. 281, 186-204 (2003)

6. Liu, S, Beretta, E, Breda, D: Predator-prey model of Beddington-DeAngelis type with maturation and gestation delays. Nonlinear Anal., Real World Appl. 11, 4072-4091 (2010)

7. Liu, Z, Yuan, R: Stability and bifurcation in a delayed predator-prey system with Beddington-DeAngelis functional response. J. Math. Anal. Appl. 296, 521-537 (2004)

8. Pang, P, Wang, M: Qualitative analysis of a ratio-dependent predator-prey system with diffusion. Proc. R. Soc. Edinb. A $133,919-942$ (2003)

9. Berezansky, L, Baštinec, J, Diblík, J, Šmarda, Z: On a delay population model with quadratic nonlinearity. Adv. Differ. Equ. 2012(1), 230 (2012)

10. Baštinec, J, Berezansky, L, Diblík, J, Šmarda, Z: On a delay population model with a quadratic nonlinearity without positive steady state. Appl. Math. Comput. 227, 622-629 (2014)

11. Liu, Q: The effects of time-dependent delays on global stability of stochastic Lotka-Volterra competitive model. Physica A 420, 108-115 (2015)

12. Gard, TC: Persistence in stochastic food web models. Bull. Math. Biol. 46, 357-370 (1984)

13. Gard, TC: Introduction to Stochastic Differential Equations. Dekker, New York (1988)

14. Liu, M, Wang, K: Persistence and extinction in stochastic non-autonomous logistic systems. J. Math. Anal. Appl. 375, 443-457 (2011)

15. Liu, M, Wang, K: Persistence, extinction and global asymptotical stability of a non-autonomous predator-prey model with random perturbation. Appl. Math. Model. 36, 5344-5353 (2012)

16. May, RM: Stability and Complexity in Model Ecosystems. Princeton University Press, Princeton (2001)

17. Liu, M, Wang, K, Wu, Q: Survival analysis of stochastic competitive models in a polluted environment and stochastic competitive exclusion principle. Bull. Math. Biol. 73, 1969-2012 (2011) 
18. Jiang, $\mathrm{D}, \mathrm{Shi}, \mathrm{N}, \mathrm{Li}, \mathrm{X}$ : Global stability and stochastic permanence of a non-autonomous logistic equation with random perturbation. J. Math. Anal. Appl. 340, 588-597 (2008)

19. Liu, M, Wang, K: Stochastic logistic equation with infinite delay. Math. Methods Appl. Sci. 35, 812-827 (2012)

20. Du, B, Wang, YM, Lian, XG: A stochastic predator-prey model with delays. Adv. Differ. Equ. 2015, Article ID 141 (2015)

21. Schreiber, SJ, Benaim, M, Atchade, KAS: Persistence in fluctuating environments. J. Math. Biol. 62(5), 655-683 (2011)

22. Liu, M, Bai, C: Analysis of a stochastic tri-trophic food-chain model with harvesting. J. Math. Biol. 73(3), 597-625 (2016)

23. Zhu, Y, Liu, M: Permanence and extinction in a stochastic service-resource mutualism model. Appl. Math. Lett. 69, 1-7 (2017)

24. Higham, DJ: An algorithmic introduction to numerical simulation of stochastic differential equations. SIAM Rev. 43(3), 525-546 (2001)

25. Liu, M, Bai, C: Dynamics of a stochastic one-prey two-predator model with Lévy jumps. Appl. Math. Comput. 284, 308-321 (2016)

26. Liu, M, Bai, C, Jin, Y: Population dynamical behavior of a two-predator one-prey stochastic model with time delay. Discrete Contin. Dyn. Syst. 37(5), 2513-2538 (2017)

27. Liu, M, Bai, C: Optimal harvesting of a stochastic delay competitive model. Discrete Contin. Dyn. Syst., Ser. B 22, 1493-1508 (2017)

\section{Submit your manuscript to a SpringerOpen ${ }^{\circ}$ journal and benefit from:}

- Convenient online submission

- Rigorous peer review

Open access: articles freely available online

- High visibility within the field

Retaining the copyright to your article

Submit your next manuscript at $\boldsymbol{\nabla}$ springeropen.com 